SYSTEMATIC REVIEW

\title{
Community based prevention programs targeting all injuries for children
}

\author{
A Spinks, C Turner, R McClure, J Nixon
}

Injury Prevention 2004;10:180-185. doi: 10.1136/ip.2003.004903

See end of article for authors' affiliations ....................

Correspondence to: Cathy Turner, Injury Research Unit, School of Population Health, Mayne Medical School, University of Queensland, Herston Rd, Herston, QLD 4006, Australia; C.Turner@ sph.uq.edu.au
Objective: Community based models for injury prevention have become an accepted part of the overall injury control strategy. This systematic review of the scientific literature examines the evidence for their effectiveness in reducing all-cause injury in children 0-14 years of age.

Methods: A comprehensive search of the literature was performed using the following study selection criteria: community based intervention study; children under 14 years; outcome measure was injury rates; and either a community control or an historical control was used in the design. Quality assessment and data abstraction were guided by a standardized procedure and performed independently by two authors. Data synthesis was in tabular and text form with meta-analysis not being possible due to the discrepancy in methods and measures between the studies.

Results: Thorough electronic and library search techniques yielded only nine formally evaluated community based all-cause child injury prevention programs that have reported actual injury outcomes. Of these nine studies, seven provided high level evidence where contemporary control communities were used for comparison; the remaining two used a pre and post-design or time trend analysis where historical data from the community were used as the comparison. Only three of the seven studies with contemporary control communities found significant effect of the intervention; the two studies without controls noted significant reductions in injury rates after the intervention period.

Conclusion: There is a paucity of research from which evidence regarding the effectiveness of community based childhood injury prevention programs can be obtained and hence a clear need to increase the effort on developing this evidence base.
S ince the development of the public health/epidemiological approach to injury control ${ }^{1}$ pioneered by Haddon ${ }^{2}$ and Gordon ${ }^{3}$ in the 1960s, models upon which interventions have been based have undergone progressive development. During the 1970s, there was a strong focus on engineering solutions and top down legislative changes. These solutions have immediate applicability and are largely effective in highly defined, single purpose public environments such as the workplace and road environment. In other areas such as childhood injury, however, environments are far more varied, less clearly delineated, largely private, and hence far less easy to engineer and regulate. Researchers in these contexts have concentrated on developing specific countermeasures which have not achieved the level of success anticipated. It was realised that simply introducing the countermeasure (for example, car seat restraints, bicycle helmets) was not sufficient, and that an effective means of conveying the countermeasure was necessary, so that it could become embedded in the social and physical structures of community function. ${ }^{4}$ On the strength of this realisation the community based (or community intervention) models for injury prevention were developed in the 1980s and 1990s and have now become an accepted part of the overall injury control strategy. ${ }^{4}$

The community based model has been characterised as having a shared ownership of the injury problem and its solution by experts and community members, and joint responsibility for determining appropriate priorities and interventions. It acknowledges a complex causal web embedded in social and organisation structures; a coordinated multistrategy response; and an emphasis on optimising community involvement. ${ }^{4}$ This model underpins a number of growing global movements, including the United States
National Safe Kids and Safe Kids Worldwide Campaigns and the World Health Organization (WHO) Safe Communities and similarly inspired national movements in other individual countries such as the Canadian Safe Communities Foundation and the Beterem National Centre for Children's Health and Safety in Israel. These programs, despite having originated in different parts of the world in order to confront unique political, environmental, and sociocultural challenges, all have similar frameworks for action in that the community is the driving impetus for injury reduction activities.

For example, the United States Safe Kids Campaign was established in 1987 and is currently comprised of approximately 500 individual chapters. The campaign acts at a community level through education via media campaigns and retail promotions; community empowerment through grassroots organisations, environmental changes, legislative enactment of safety regulations and standards; and evaluative research and injury surveillance. The global campaign, Safe Kids Worldwide Campaign was inspired by the United States campaign and established in 2000 with 14 member countries.

As a second example, the WHO Safe Communities concept was formally initiated in 1989 as a response to a successful pilot project in Sweden in which a $23 \%$ decrease in total population injury rates was achieved. ${ }^{6}$ The Safe Communities ideology stipulates that safety can be achieved through integrated, collaborative efforts that are implemented in a supportive social, cultural, and political environment and that community members play the leading role for injury prevention. ${ }^{7}$ Currently, there are 78 formally designated WHO Safe Communities and 11 Affiliated Support Centres around the world, with many more communities under preparation to meet the WHO Safe Community criteria. Many of these 


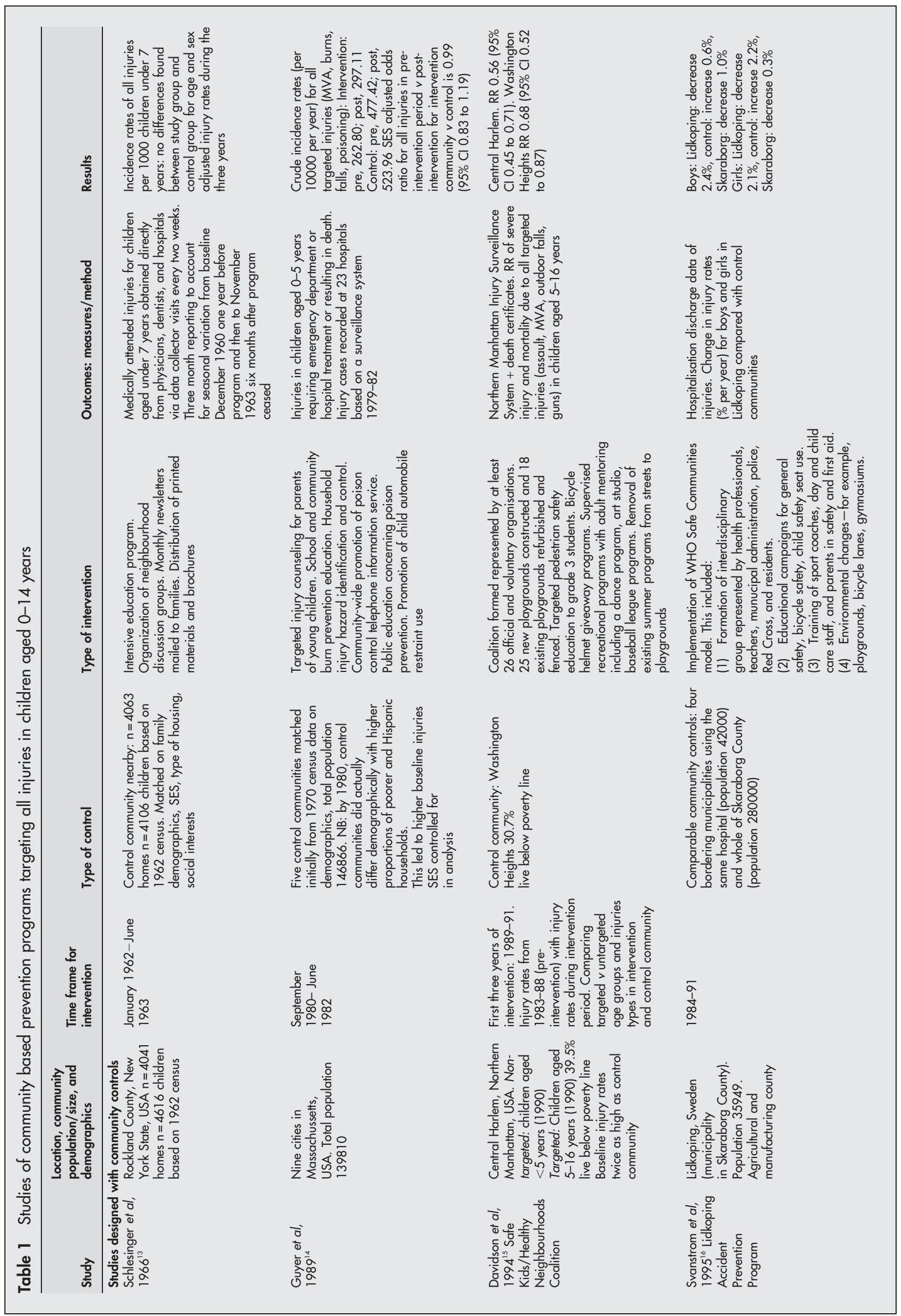




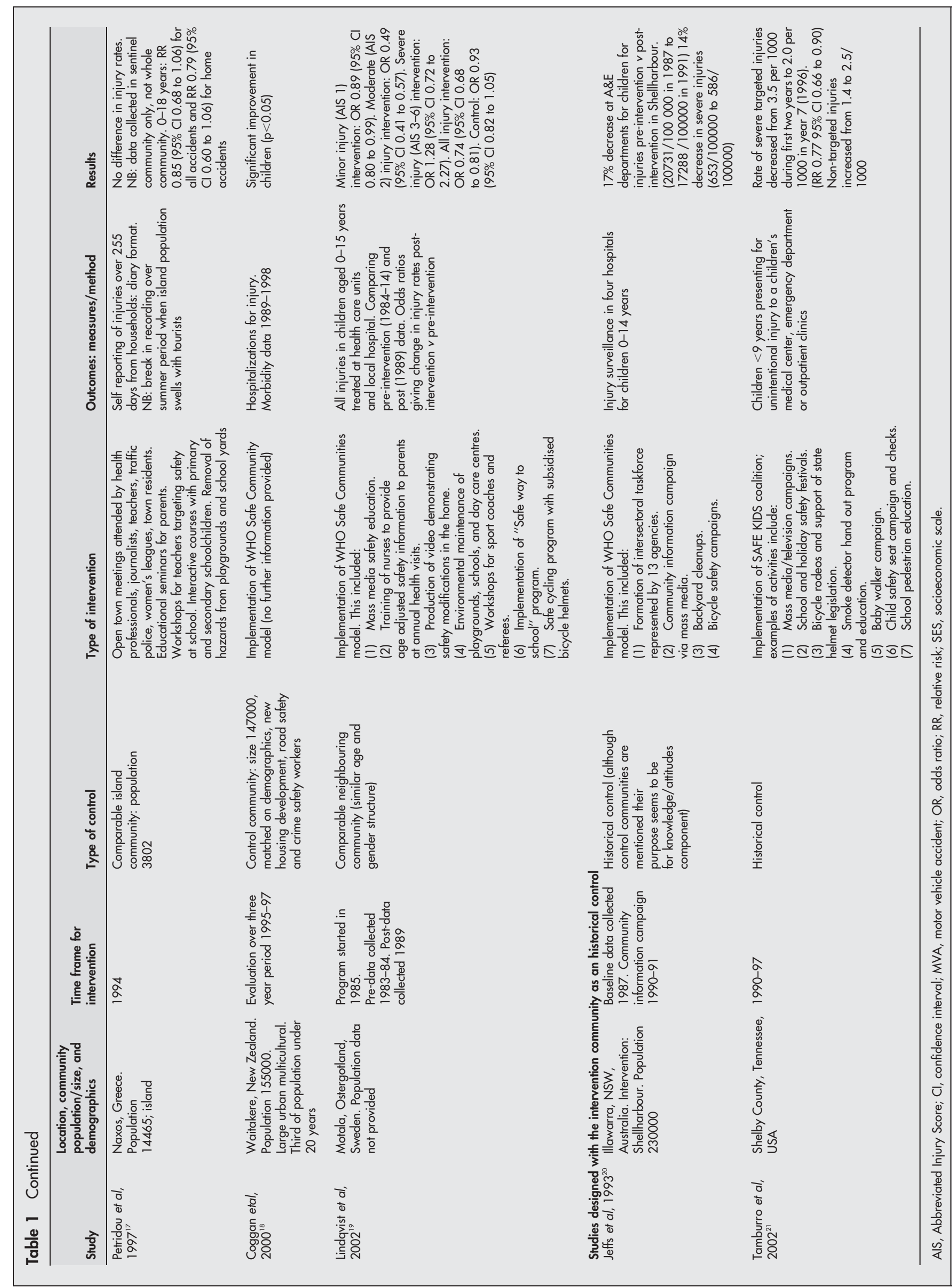

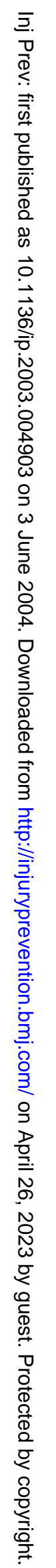


Safe Communities target childhood injury specifically as part of their program activities.

Characteristic of community based prevention strategies is the devolving of ownership, responsibility, and costs from centralised government departments back to the community. Justification for the substantial imposition on the community is that these multistrategy multifocused, community based childhood prevention programs are effective in reducing the frequency and severity of injury in children. The aim of this paper is to test this hypothesis by systematically reviewing the relevant evidence in the literature.

\section{METHODS}

An electronic search for published studies was conducted using WebSPIRS as a search tool for Medline (1966-2003), CINAHL (1982-2002), and PsycINFO (1872-2003) databases. The following search strategy was used:

(child* OR adolescent OR pediatric OR paediatric) AND (strateg* OR intervention* OR program* OR prevent*) AND (injur* OR wound* OR trauma OR fracture*) AND (community OR communities OR population)

The initial deliberately broad search strategies yielded over 22000 studies based on child injury prevention related search terms. When the search was limited to look at studies conducted within a community or population setting, the combination of the search terms above yielded 3441 abstracts from Medline, 3261 abstracts from CINAHL, and 545 abstracts from psycINFO. Based on examination of these titles and abstracts, full texts for 35 studies were retrieved.

Futher potential studies were identified by screening the reference lists of existing systematic reviews on this topic. ${ }^{8-11}$ Separate hand and electronic searches of the journals Injury Prevention (1995-2003) and Accident Analysis and Prevention (1995-2003) were performed, however no further articles were identified. A key word search of EMBASE (1992-2003) and the Cochrane library did not reveal any suitable literature.

After closer examination of the full text of the retrieved studies by the first author, a large number were discarded as they either lacked an evaluation component or clearly did not meet the definition for a community based intervention. The second and third authors independently examined the remaining 12 as per the following study selection criteria:

(A) Community based intervention study; defined as an intervention that applies more than one single strategy and is targeted towards a whole community or group of individuals therein.

(B) Target population is children under 14 years.

(C) Outcome measure is injury rates from all causes.

(D) Community control or historical control is used in the design.

Nine studies met all the inclusion criteria for this review and were subject to a further data extraction process conducted independently by the second and third authors with the first author acting to reconcile differences. This process was guided by a standardized abstraction procedure developed to improve the evaluation of the quality of studies selected in a review. ${ }^{12}$ The form constructed for the purpose of this review is available on request. Each study was independently assessed for the quality of the execution of the study by the second and third authors. The results of the data abstraction process are outlined in table 1 .

\section{RESULTS}

Seven studies were designed with community controls for comparison with the intervention community. ${ }^{13-19}$ A further two studies were designed using the intervention community as an historical control in a before-after design. ${ }^{20-21}$ Metaanalysis was not possible due to the diversity of the interventions and the outcome measures reported in each of the studies reviewed. Three studies that were screened by the second and third authors were excluded. ${ }^{22-24}$ The reasons for exclusion were: the paper described part of a larger study ${ }^{14}$ already included for analysis ${ }^{22}$; no injury outcomes were evaluated $^{23}$; and the study did not meet definition for a community based intervention. ${ }^{24}$

\section{Studies evaluating a community based intervention using a control community design}

Schlesinger et al evaluated the impact of a childhood injury prevention project implemented in Rockland County, New York State that focused on community based education of self selected groups. ${ }^{13}$ Injury rates for children under 7 years were compared for those families exposed to the project interventions to a comparable group of non-exposed families. Injury incidence rates for children under 7 years were assessed every three months for 12 months before, during, and six months after the project. No differences were found between the study group and the control group for age and sex adjusted injury rates or injury severity. The authors note the importance of continued reporting for a sustained period of time when evaluating community programs, particularly post-program, to ensure valid conclusions are derived.

Guyer et al report on a statewide community based injury prevention program designed to reduce the incidence of burns, falls in the home, motor vehicle occupant injuries, poisoning, and suffocations in $0-5$ year olds. ${ }^{14}$ The intervention was implemented in nine cities in Massachusetts with the baseline and post-program injury incidence rates of these communities compared with those obtained from five control communities. While the control communities were originally matched demographically based on census data their demographic distribution changed during the time of the study to include higher proportions of poorer Hispanic households. Results were stratified and analyzed according to three levels of median family income and while there was a significant reduction in motor vehicle occupant injuries, no association was found between the intervention and a reduction in any of the other targeted injuries post-campaign. An effort was made to assess the outreach of the program with the authors estimating about $42 \%$ of households were exposed to one or more interventions over the two year period. Statewide legislation for child auto restraints was being debated at the time of the study and may partially explain why a positive finding was found only for motor vehicle occupant injuries; study results indicate that the level of child auto restraint use rose in both the intervention and control communities postcampaign.

A Safe Kids/Healthy Neigbourhoods coalition in Central Harlem was formed in 1989 initially to target a reduction in outdoor falls for children aged 5-16 years. ${ }^{15}$ Over the next three years community activities expanded to include targeting a reduction in traffic accidents, assaults, and firearms for the same age group and then compare the injury incidence for these causes in Central Harlem to the incidence in a nearby health district, Washington Heights that received no intervention. Injury outcomes data were obtained from a surveillance system operating in two major hospitals that served both communities. There was an overall decline in injuries for the age group and outcomes targeted in the intervention community, however a similar decline also occurred in the control community. The risk of outdoor falls actually increased in the intervention community with the authors suggesting that the program led to an increase in supervised sporting activities for school aged children and that the outcome measure used could not adequately 
distinguish between indoor and outdoor falls due to classification coding. There was a specific reduction in the incidence of assault and gun injuries in the intervention community that did not occur in the control community, however there was an equal reduction in motor vehicle injuries across both communities. The authors acknowledge a secular trend and/or spillover effects as possible reasons for their mixed findings.

Svanstrom et al report on the impact of the safe community intervention targeting childhood injuries in Lidkoping, Sweden. ${ }^{16}$ Injury incidence rates in the intervention community were compared over the nine year study period with injury incidence in four bordering municipalities and against the injury incidence for the whole of the county. Results indicate an average annual decrease of $2 \%$ over the nine years for both sexes in the intervention community and an average annual increase for boys of $0.6 \%$ and for girls of $2 \%$ in the neighboring control communities. There is no discussion of similarities or differences between Lidkoping and the four control municipalities to determine if these small variations in rates could be explained by other confounding factors. While the linear regression analysis provides an average annual percentage change, it is not clear whether this analysis was adjusted for secular trends occurring before or during the intervention.

A health education injury prevention program was implemented and evaluated on one Greek island with another Greek island serving as a control community that received no intervention. ${ }^{17}$ The program targeted home injuries for children aged less than 18 years. The study was very small, relied on self report for injury outcomes, and did not show a significant reduction in injuries as a result of the program. Study outcomes were also not measured during the summer months due to the impact of tourism in both communities. The authors suggest that large scale national injury prevention programs seem to be more successful in reducing injuries rather than specific small scale intervention projects similar to their own study.

An evaluation of the Waitakere Community Injury Prevention Project is reported by Coggan et al. ${ }^{18}$ While the program was based on the WHO safe community model and targeted all ages, childhood injuries were a specific focus and separate analysis is reported for the 0-14 year age group. Significant reductions in injury incidence for children were found in the two years after the program with corresponding increases in the comparison community. One limitation of the study is the lack of detail in reporting the findings, only a $\mathrm{p}$ value is reported based on an unknown test to determine significant changes in the regression slopes pre-intervention and post-intervention. Incidence rates are reported graphically with trends indicating an increase in incidence from 1989 for the intervention community, the comparison community and the whole of Auckland.

The impact of a WHO Safe Community model on the incidence of injuries for children aged 0-15 years was evaluated by Lindqvist et al. ${ }^{19}$ The safe community of Motala, Sweden was assigned a control community for comparison of minor, moderate, and severe injuries of all types in children. Results indicate a post-program reduction in injuries for all children aged 0-15 years of $26 \%$ in the intervention community and a 7\% non-significant reduction in the control community exposed only to national prevention programs. Detailed data on minor, moderate, and severe injuries are not provided for the control community and the population size of each community is not given. Crude injury counts provided suggest the control community was much smaller than the intervention community, which may also explain the lack of statistical significance for the odds ratio calculated to determine an effect post-program in injury rates. It is not clear whether pre-program and post-program data on injury rates were collected using the same methods, which could be a serious limitation to the study findings. Firstly, pre-program injury rates differed between the control and intervention communities which the authors explain was due to the difference in the proportions of each population seeking medical care from the one hospital used to collect outcome data. Secondly, changes in injury rates are only provided for the intervention community. More information is required in order to draw valid conclusions from this study design.

\section{Studies evaluating a community based intervention using an historical control design}

The impact of another WHO Safe Community model implemented in an Australian city to reduce childhood injuries is reported by Jeffs et al. ${ }^{20}$ Results indicate a $17 \%$ decrease in childhood injuries pre-intervention to postintervention, however the authors note variability across four hospitals in reporting outcome data during the study period and additional under-reporting of injury rates for children treated outside of a hospital. It is difficult to solely attribute the reduction in injury rates to the intervention program without a comparison community and an attempt to control for other confounding factors.

Tamburro et al assessed the association between the United States National Safe Kids Coalition and injury rates in one county 10 years after the campaign originated..$^{21}$ Outcome data were based on severe injuries presenting to the only children's hospital in the county from 1990-97. After controlling for secular trends in hospital admission rates and demographics, a $23 \%$ reduction for males was noted but no significant reduction for females was found. The authors note the limitations of their study design, particularly the lack of control community for comparison and the impact of potential confounders that might otherwise explain the association found.

\section{DISCUSSION}

It is unknown how many community based childhood prevention programs have or are being currently implemented across the globe, however, in just the Safe Kids and Safe Communities networks in North America there are over 300 registered activities. Of all the community based child injury prevention programs throughout the world, we could only find nine whose formal evaluations against injury outcomes have been reported in the scientific literature. Of these nine studies, seven provide high level evidence where contemporary control communities were used for comparison with the remaining two using a pre and post-design or time trend analysis where historical data from the community were used as the comparison. Only three of the seven studies with contemporary control communities found a significant effect of the intervention, with the two studies without controls noting significant reductions in injury rates after the intervention period. There are insufficient studies and too great a variation in the results to provide definitive evidence as to the effectiveness or otherwise of community based, multistrategy multifocused programs for the prevention of all cause injury in children.

Strengths of this review are that it collects for the first time the evidence base justifying what has become a widely practised form of prevention and enables this evidence to be judged on its merits. Limitations in the methodology of this review are unlikely to have influenced the findings. Positive publication bias may have increased the proportion of papers reporting positive results and difficulty ensuring a completely comprehensive ascertainment of relevant published papers may have led to randomly distributed omissions. Key papers, however, 


\section{Key points}

- Community based models for injury prevention have become an accepted part of the overall injury control strategy.

- There is a paucity of research studies in the literature from which evidence regarding the effectiveness of community based childhood injury prevention programs can be obtained.

- A systematic review of the literature found only nine studies of community based injury prevention programs that included an evaluative component, seven that used a trial design with a contemporary control.

- High quality evaluations of community based injury prevention programs are required to determine if these programs achieve population level improvements in injury outcomes.

will have been referred to in references of the recent articles and some level of confidence in the completeness of the search of at least the peer reviewed literature can be accepted.

The way forward is to identify those elements that distinguish successful community based injury prevention programs. In order for this to be achieved, the evidence base must be broadened to include a wider variety of properly evaluated community based injury prevention programs. This will then enable a more in-depth interpretation of the mediating environmental, cultural, geographical, and political influences that shape communities around the world and impact on population level injury rates.

\section{IMPLICATIONS FOR PREVENTION}

There is a paucity of studies in the literature from which evidence regarding the effectiveness of community based childhood injury prevention programs can be ascertained and hence a clear need to increase the effort on developing this evidence base. Formal evaluations should be expected of any new programs undertaken. However sufficient evidence does exist to continue to undertake this form of prevention program pending definitive collation of an evidence base to direct their form and function.

\section{ACKNOWLEDGEMENTS}

The research reported in this publication is a project of Injury Prevention and Control (Australia) Ltd (http://www.ipca.com.au/), supported by a grant from the National Health and Medical Research Council.

\section{Authors' affiliations}

A Spinks, Injury Research Unit, School of Population Health, Mayne Medical School, University of Queensland, Brisbane, Australia
C Turner, Injury Research Unit, School of Population Health, Mayne Medical School, University of Queensland, Brisbane, Australia

R McClure, Injury Research Unit, School of Population Health, Mayne

Medical School, University of Queensland, Brisbane, Australia

J Nixon, Department of Paediatric and Child Health, School of

Medicine, University of Queensland, Brisbane, Australia

\section{REFERENCES}

1 Kraus JF, Robertson LS. Injuries and public health. In: Last JM, Wallace RB, eds. Public health and preventive medicine. Connecticut: Appleton and Lange, 1992:1021-34.

2 Haddon WJ. A note concerning accidental theory and research with special reference to motor vehicle accidents. Ann N Y Acad Sci 1963;107:635-46.

3 Gordon J. The epidemiology of accidents. Am J Public Health 1949:39:504-15

4 Moller J. Community-based interventions: an emerging dimension of injury control models. Health Promotion Journal of Australia 1991;1(2):51-4.

5 Christoffel T, Gallagher SS. Injury prevention and public health. Gaithersburg, MD: Aspen Publishers, 1999.

6 Schelp L. Epidemiology as a basis for evaluation of a community intervention programme on accidents (dissertation). Sundyberg, Sweden: Karolinska Institute, 1987.

7 Manifesto for Safe Communities. Safety-a universal concern and responsibility for all. First World Conference on Accident and Injury Prevention; 1989. Geneva: World Health Organization, 1989.

8 Klassen TP, MacKay JM, Moher D, et al. Community-based injury prevention interventions. Future Child 2000; 10:83-110.

9 Towner E, Dowswell T, Mackereth C, et al. What works in preventing unintentional injuries in children and young adolescents? An updated systematic review. London: NHS. Health Development Agency, 2001.

10 Dowswell T, Towner EM, Simpson G, et al. Preventing childhood unintentional injuries - what works? A literature review. Inj Prev 1996;2:140-9.

11 Dowswell T, Towner E. Social deprivation and the prevention of unintentional injury in childhood: a systematic review. Health Educ Res 2002;17:221-37.

12 Zaza S, Wright-De Aguero LK, Briss PA. Data collection instrument and procedure for systematic reviews in the Guide to Community Preventive Services. Am J Prev Med 2000;18:44-74.

13 Schlesinger ER, Dickson DG, Westaby J, et al. A controlled study of health education in accident prevention: the Rockland County Child Injury Project. 1966. Inj Prev 1997;3:218-23.

14 Guyer B, Gallagher SS, Chang BH, et al. Prevention of childhood injuries: evaluation of the Statewide Childhood Injury Prevention Program (SCIPP). Am J Public Health 1989;79:1521-7.

15 Davidson LL, Durkin MS, Kuhn L, ef al. The impact of the Safe Kids/Healthy Neighborhoods Injury Prevention Program in Harlem, 1988 through 1991. Am J Public Health 1994;84:580-6.

16 Svanstrom L, Ekman R, Schelp L, et al. The Lidkoping Accident Prevention Programme-a community approach to preventing childhood injuries in Sweden. Ini Prev 1995;1:169-72.

17 Petridou E, Tolma E, Dessypris N, et al. A controlled evaluation of a community injury prevention project in two Greek Islands. Int J Epidemiol 1997;26:173-9.

18 Coggan C, Patterson P, Brewin M, et al. Evaluation of the Waitakere Community Injury Prevention Project. Inj Prev 2000;6:130-4.

19 Lindqvist K, Timpka T, Schelp L, et al. Evaluation of a child safety program based on the WHO Safe Community Model. Inj Prev 2002;8:23-6.

20 Jeffs $D$, Booth $D$, Calvert D. Local injury information, community participation and injury reduction. Aust J Public Health 1993;17:365-72.

21 Tamburro RF, Shorr RI, Bush AJ, et al. Association between the inception of SAFE KIDS coalition and changes in pediatric unintentional injury rates. Ini Prev 2002;8:242-5.

22 Bass JL, Mehta KA, Ostrovsky M. Childhood injury prevention in a suburban Massachusetts population. Public Health Rep 1991;106:437-42.

23 Kemp A, Gibbs N, Vafidis G, et al. Safe Child Penarth: experience with a Safe Community strategy for preventing injuries to children. Inj Prev 1998;4:63-8

24 Nicholson AJ, Duff S, Ryan M, et al. The use of local accident and emergency injury surveillance to monitor the impact of a lay safety community programme. Ir Med J 2002;95:143-5. 